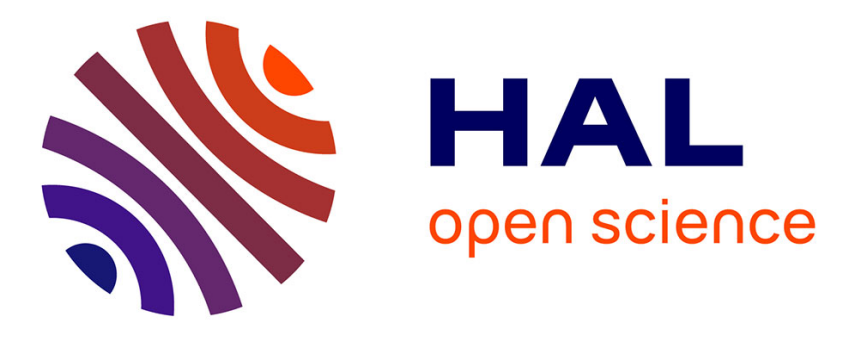

\title{
4DGVF segmentation of vector-valued images
}

Vincent Jaouen, Paulo Gonzalez, Sylvie Chalon, Denis Guilloteau, Irène

Buvat, Clovis Tauber

\section{To cite this version:}

Vincent Jaouen, Paulo Gonzalez, Sylvie Chalon, Denis Guilloteau, Irène Buvat, et al.. 4DGVF segmentation of vector-valued images. IEEE International Conference in Image Processing, Oct 2014, Paris, France. pp.1. hal-01064954

\section{HAL Id: hal-01064954 https://hal.science/hal-01064954}

Submitted on 17 Sep 2014

HAL is a multi-disciplinary open access archive for the deposit and dissemination of scientific research documents, whether they are published or not. The documents may come from teaching and research institutions in France or abroad, or from public or private research centers.
L'archive ouverte pluridisciplinaire HAL, est destinée au dépôt et à la diffusion de documents scientifiques de niveau recherche, publiés ou non, émanant des établissements d'enseignement et de recherche français ou étrangers, des laboratoires publics ou privés. 


\title{
4DGVF SEGMENTATION OF VECTOR-VALUED IMAGES
}

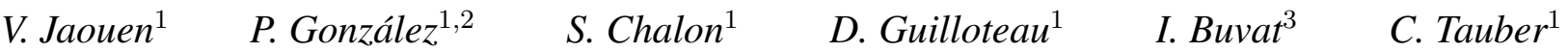 \\ ${ }^{1}$ UMR INSERM U930 «Imagerie et cerveau», Université François-Rabelais de Tours \\ 2 bd Tonnelé, 37044 Tours Cedex, France \\ ${ }^{2}$ Universidad Católica del Maule, Talca, Chile \\ ${ }^{3}$ SHFJ/CEA, Orsay, France
}

\begin{abstract}
In this paper, we extend the gradient vector flow field to the vector-valued case for robust variational segmentation of 4D images with active surfaces. Instead of only exploiting scalar edge strength in order to identify vector edges, we propagate both directions and amplitudes of vector gradients computed from the analysis of a structure tensor of the vector-valued image. To reduce contributions from noise in the calculation of the structure tensor, image channels are weighted according to a blind estimator of contrast that take profit of the deformable models framework. The proposed 4DGVF vector field is validated on synthetic image datasets and applied to biological volume delineation in dynamic PET imaging.
\end{abstract}

Index Terms - Deformable models, Image segmentation, Gradient vector flow, Vector-valued images, Positron emission tomography

\section{INTRODUCTION}

Active surface models attempt to recover a region of interest by conforming to its boundary an evolving surface superimposed onto the image domain $[1,2]$. The evolution of the surface toward equilibrium can be seen as a forcebalance relation between forces acting on the surface : internal forces, which control the smoothness of the model, and external forces, derived from image information. In this paper, we focus on edge-based parametric deformable models. Among external force fields used for parametric models, gradient vector flow $(\mathrm{GVF})$ fields $[4,5]$ aroused great interest due to their reduced sensitivity to noise and their ability to progress into concave regions in the image. However, their extension to the vector-valued case is not straightforward.

It is generally not satisfying to average the gradient orientations of the different channels in order to express the gradient of a vector-valued image, or vector gradient [6]. A more relevant approach was proposed by Di Zenzo who considered the image as a vector field [7]. In this context, the gradient

The research leading to these results has received funding from the European Union's Seventh Framework Programme (FP7/2007-2013) under grant agreement HEALTH-F2-2011-278850 (INMiND). direction and amplitude are associated with the maximization of a quadratic form of the total differential of the image, a problem which can be expressed algebraically using the local structure tensor (LST) formalism [8]. Previous segmentation approaches that rely on this paradigm use the amplitude of the vector gradient to identify vector edges $[9,10,11,12]$. However, relevant directional information can also be drawn from the analysis of the LST in order to further enhance gradient identification, and hence segmentation results. Moreover, the different channels generally participate equally in the calculus of the gradient, even those where the feature of interest is badly represented.

The aim of this work is to propose a new external force field for parametric deformable surfaces evolving in vectorvalued images. We introduce a new gradient vector flow field, the 4DGVF field (Four Dimensional Gradient Vector Flow) that takes advantage of both direction and amplitude of vector gradients to propagate them throughout the image. The vector gradients are drawn from the analysis of a LST of the image that combines gradient information available in the different channels. We focus our study on imaging modalities where detection is hampered by varying contrast and noise over the channels, but where physical edges locations do not vary. In such situations, while image edges may be ill-defined on individual channels, the combination of information along the different channels may improve edge location. By weighting the structure tensor dynamically, we control the influence of the different channels in order to favor the ones where the features can be better detected, hence reducing sensitivity to noise.

We assess the quality of our model using synthetic images, realistic Monte Carlo simulations of dynamic Positron Emission Tomography (dPET) images and apply it to real dPET images. dPET images consist of subsequent acquisitions of the same field of view at different time intervals and can therefore be considered as vector-valued images. We compare our results to single-channel approaches based on Generalized GVF [5] and Vector Field Convolution (VFC) [13], and to the Color GVF model of Yang et al. for vectorvalued images [11]. 


\section{PROPOSED METHOD}

\subsection{Vector geometry}

In the continuous domain, we denote by $\mathbf{I}$ a $3 \mathrm{D}$ vectorvalued image constituted of $M$ channels :

$$
\mathbf{I}(\mathbf{x}, c):\left(\Omega_{s} \otimes \Omega_{c}\right) \in \mathbb{R}^{3} \otimes \mathbb{N} \rightarrow \mathbb{R},
$$

where $\Omega_{s}$ is the $3 \mathrm{D}$ spatial domain of the image and $\Omega_{c}$ the channel dimension. $\mathbf{x}=\left(x_{1}, x_{2}, x_{3}\right) \in \Omega_{s}$ is the spatial position of the voxel. We denote by $I_{k}$ the $k^{t h}$ channel of the image. A parametric active surface $\mathbf{S}^{\tau}$ at time $\tau$ of its deformation is represented as a mapping of a bivariate parameter $(m, n)$ on a regular grid $\Omega$, superimposed on the spatial image domain :

$$
(m, n) \rightarrow \mathbf{S}^{\tau}(m, n)=\left[x_{1}(m, n), x_{2}(m, n), x_{3}(m, n)\right]^{T} .
$$

The vector gradient is associated with the direction that maximizes the quadratic form of the total differential $d \mathbf{I}$ of $\mathbf{I}$ [7]. In the $3 \mathrm{D}$ case, $\mathbf{I}$ is a $3 \mathrm{D} \rightarrow M \mathrm{D}$ vector field and a weighted quadratic form, or first fundamental form of $d \mathbf{I}$ is expressed as :

$$
\|d \mathbf{I}\|_{\omega}^{2}=d \mathbf{x}^{T} \mathbf{G}_{\omega} d \mathbf{x}
$$

with $G_{\omega}$ a regularized, weighted LST [14] :

$$
G_{\omega}=K_{\sigma} * \sum_{k=1}^{M} \omega_{k}\left(\nabla I_{k} \otimes \nabla I_{k}^{T}\right),
$$

where $K_{\sigma}$ is a Gaussian kernel of scale $\sigma, *$ is the convolution operation, $\otimes$ is the tensor product, and $\omega_{k}$ is a weighting factor for channel $\mathbf{I}_{k}$. The weighting factors $\omega_{k}, k \in\{1 . . M\}$ allow to characterize the reliability of the different channels. Without a priori knowledge, the extension of Di Zenzo's approach to $3 \mathrm{D}$ consists in weighting all channels equally by choosing $\omega_{k}=1 / M, \forall k$. With such weights, contributions from noise in channels where the object is poorly represented might hamper edge detection. Taking profit of the deformable model framework, we propose a blind estimation of the weights based on the object representativeness in the channels. For each channel $\mathbf{I}_{k}$, let $R_{k}^{i n}$ be the set of voxels located inside $\mathbf{S}^{t}$, and let $R_{k}^{\text {out }}$ be the set of voxels located outside $\mathbf{S}^{t}$ and inside $\lambda \mathbf{S}^{t}$, a homothetic transformation of $\mathbf{S}^{t}$ of parameter $\lambda$. The limitation of $R_{k}^{\text {out }}$ to $\lambda \mathbf{S}^{t}$ prevents possible influence from further regions. We compare the intensities of voxels located inside the region to voxels of its outer neighborhood in order to define a contrast measure. We define the weighting factor for channel $\mathbf{I}_{k}$ as follows :

$$
\omega_{k}:=\left(\frac{\left|\overline{\mathbf{I}}_{k}^{\text {in }}-\overline{\mathbf{I}}_{k}^{\text {out }}\right|^{\gamma}}{\sum_{j=1}^{M}\left|\overline{\mathbf{I}}_{j}^{\text {in }}-\overline{\mathbf{I}}_{j}^{\text {out }}\right|^{\gamma}}\right),
$$

where summation is over the $M$ channels. $\overline{\mathbf{I}}_{k}^{\text {in }}$ and $\overline{\mathbf{I}}_{k}^{\text {out }}$ are the average intensities in $R_{k}^{i n}$ and $R_{k}^{\text {out }}$ respectively. $\gamma$ is a parameter that controls the linearity of the influence of the channels. Figure 1 contains an illustration of the weighting scheme on a conceptual 2D example. Figure 1a displays a high contrast channel where average intensities $R_{1}^{\text {in }}$ and $R_{1}^{\text {out }}$ are significantly different and for which the corresponding weight would be large. On the contrary, Figure 1b shows a low contrast channel where the corresponding weight would be low.

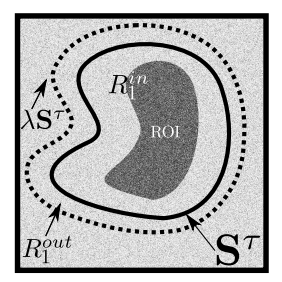

(a)

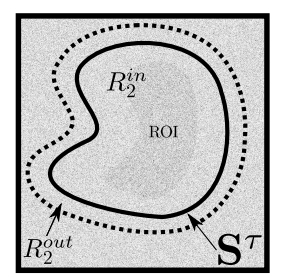

(b)
Fig. 1. Illustration of the weighting method on a $2 \mathrm{D}$ representation of the active surface $\mathbf{S}^{t}$ around a region of interest (ROI). (a) High contrast channel. (b) Low contrast channel.

\subsection{DGVF-based active surface}

\subsection{1. $4 D G V F$ external force field}

The eigenvalues $\lambda^{+}>\lambda_{1}^{-}>\lambda_{2}^{-}$of the LST $G_{\omega}$ give the scalar rates of change of the first fundamental form in a local basis of extremal variations. Depending on the applications, these eigenvalues can be combined to define different norms of the LST $[15,16,17,18]$. In our study, we choose a coherence norm that measures the amount of local anisotropy [18], a generalization to the $3 \mathrm{D}$ case of the norm presented by $\mathrm{Sa}-$ piro in [16]. This measure exhibits oriented gradient patterns in the image :

$$
\|d \mathbf{I}\|_{\omega}^{2}=\sqrt{\left(\lambda^{+}-\lambda_{1}^{-}\right)^{2}+\left(\lambda^{+}-\lambda_{2}^{-}\right)^{2}+\left(\lambda_{1}^{-}-\lambda_{2}^{-}\right)^{2}} .
$$

We define the gradient amplitude $N_{\omega}$ as : $N_{\omega}=\|d \mathbf{I}\|_{\omega}$. The directions of the eigenvectors of $G_{\omega}$ give the directions of local extrema of the quadratic form (2). The eigenvector $\vec{\theta}_{+}$associated with the maximum eigenvalue $\lambda_{+}$gives the gradient direction, and the other two define orthogonal "isophote" directions. We propose here, rather than only exploiting the eigenvalues of the LST $[9,11,12]$, to also take advantage of the directional information carried by $\vec{\theta}_{+}$. We define a vectorial edge map $\overrightarrow{\mathbf{V}}$, a vector field collinear to the local dominant eigenvectors of $G_{\omega}$, but oriented toward the nearest vector edge :

$$
\overrightarrow{\mathbf{V}}=\vec{\theta}_{+} \operatorname{sign}<\vec{\theta}_{+}, \nabla N_{\omega}>
$$

where $<,>$ denotes the dot product. 
The 4DGVF external force field is the result of nonlinear diffusion of the vectorial edge map $\overrightarrow{\mathbf{V}}$ throughout the image. The 4DGVF field is defined as the steady-state solution of the following vector partial differential equation :

$$
\frac{\partial \overrightarrow{\mathbf{F}}_{e x t}}{\partial t}=g\left(N_{\omega}\right) \Delta \overrightarrow{\mathbf{F}}_{e x t}-h\left(N_{\omega}\right)\left(\overrightarrow{\mathbf{F}}_{e x t}-\overrightarrow{\mathbf{V}}\right),
$$

where $g(s)=\mathrm{e}^{-|\nabla s| / \kappa}$ and $h=1-g$ are two functions that control the tradeoff between the first and second terms through parameter $\kappa$ [5], and $\Delta$ is the vector Laplace operator. In the vicinity of vector edges, as measured by $N_{\omega}$, the directions of $\overrightarrow{\mathbf{F}}_{\text {ext }}$ are constrained by $\overrightarrow{\mathbf{V}}$, while isotropic diffusion of $\overrightarrow{\mathbf{F}}_{\text {ext }}$ prevails in homogeneous regions. We obtain a regularized vector field oriented locally toward the nearest vector edge.

\subsubsection{Model deformation}

At each iteration $\tau$ of the deformation, the surface $\mathbf{S}^{\tau}$ undergoes locally the external force field $\overrightarrow{\mathbf{F}}_{\text {ext }}$. To avoid convergence issues and ensure a smooth deformation, the deformation force field is projected on the normal direction to $\mathbf{S}^{\tau}$. The surface is iteratively moved according to the following EulerLagrange equation :

$$
\frac{\partial \mathbf{S}^{\tau}}{\partial \tau}=\alpha \Delta \mathbf{S}^{\tau}-\beta \Delta^{2} \mathbf{S}^{\tau}+<\overrightarrow{\mathbf{F}}_{e x t}, \vec{n}>
$$

where elasticity terms are weighted by $\alpha$ and rigidity terms by $\beta$. $\vec{n}$ denotes the normal direction to the local surface element $d \mathbf{S}^{\tau}$. The LST is computed according to the proposed weighting scheme, and at each timestep $\tau$, weights are recomputed to construct a more accurate external force field for the next iteration. Internal energy parameters were set for all methods to typical values found in the literature $\alpha=0.2$ and $\beta=1.0$.

\subsection{Initialization}

In general, the energy landscape associated with a variational segmentation problem is not convex, requiring the initial model to be close to the desired optimum [19]. To this end, we propose to initialize the 4DGVF model with the Poisson Inverse Gradient algorithm [20] adapted here to the vectorvalued case. We build an initialization field $\overrightarrow{\mathbf{F}}_{\text {ext }}^{0}$ based upon the 4DGVF framework for which we use equal weighting of all channels, as finer weights such as proposed in eq. (4) can only be derived after an initial surface is defined. Once $\overrightarrow{\mathbf{F}}_{\text {ext }}^{0}$ is computed, we estimate the scalar potential $E_{\text {ext }}$ by solving the Poisson equation :

$$
\Delta E_{e x t}=-\nabla \cdot \overrightarrow{\mathbf{F}}_{e x t}^{0},
$$

This equation is solved numerically by matrix inversion for which Dirichlet boundary conditions are applied on the boundary of the image domain. We scale $E_{\text {ext }}$ in the range
$[0,-1]$, and perform $P$ energy isosurface reconstructions $E_{p}=\left(E_{1}, \ldots, E_{P}\right), E_{p} \in[0,-1]$, using a marching cubes algorithm [21]. We then select the closed model with minimal total external energy. This shape is then used for the computation of the initial weights prior to the deformation.

\section{RESULTS}

The algorithm was implemented using MATLAB using a finite difference scheme. We assessed the proposed 4DGVF active surface model by comparing it with three other models of the literature :

1) Generalized Gradient Vector Flow (GGVF) active surface model [5]. The diffusion of the gradient vectors was performed on a channel-by-channel basis, where the edge map of each channel $I_{k}$ was defined as $f_{k}=K_{\sigma} *\left|\nabla I_{k}\right|$;

2) Vector Field Convolution (VFC) active surface model [13]. A convolution was performed on a channel-by-channel basis between $f_{k}$ and a vector field kernel $\vec{C}$ in which all vectors point toward the kernel's center : $\overrightarrow{\mathbf{F}}_{e x t}=\vec{C} * f_{k}$. As performances of the two above models depended on the channel studied, we retained the result produced in the channel with best Jaccard index value.

3) Color Gradient Vector Flow (CGVF) active surface model [11]. This vector-valued model computes a single edge map based on Di Zenzo's vector gradient amplitude, wherein all channels are considered equally for the calculation of the LST. On the contrary of GGVF and VFC, a single active surface result was thus obtained for each dynamic image with both CGVF and 4DGVF approaches.

For all methods, the parameters were established so as to maximize the Jaccard similarity criterion [22] between the segmentation result and the ground truth. All models were initialized using the same surface model to prevent results from being affected by this step.

\subsection{Synthetic images}

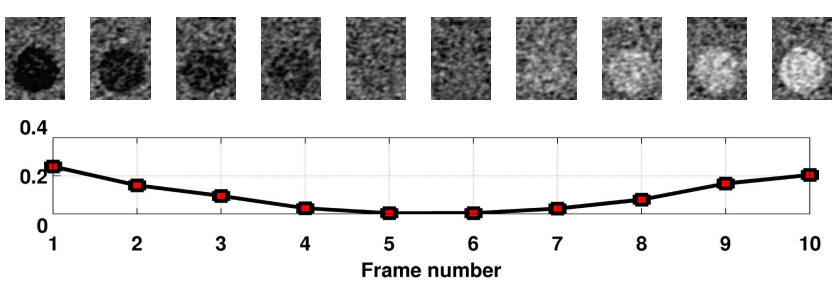

Fig. 2. Top : 2D slices of the 10 channels of a synthetic $4 \mathrm{D}$ image that exhibits varying contrast with background. Bottom : corresponding 4DGVF weights obtained for this image.

A set of images of $70 \times 50 \times 40 \times 10$ voxels was generated for which the contrast of the object with the background varied along the channels. The different channels of one of 
(a)

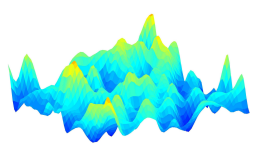

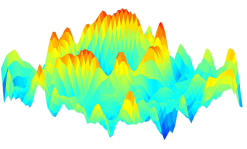

(b)

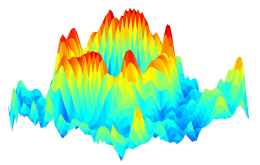

(c)
Fig. 3. Surface plots of the gradient amplitude of a synthetic 4D image : (a) $f_{k}$ (best contrasted channel), (b) with equal weighting of all channels, (c) with proposed weighting scheme

these synthetic 4D images are displayed in the top row of figure 2, where 2D slices are shown for the sake of readability. The bottom row of figure 2 displays the corresponding weights obtained with the proposed 4DGVF approach, which are consistent with the observed variations of contrast.

In order to study the influence of weighting the LST, we compared the gradient amplitude $f_{k}$ obtained in the best contrasted channel $I_{k}$ (Fig. 3a) to the vector-gradient amplitude obtained with equal weighting of all channels (Fig. 3b) and to the proposed weighted vector gradient amplitude $N_{\omega}$ (Fig. 3c). In this image, the proposed weighting scheme led to an enhancement of the edge signal while, in flat regions, spurious variations due to noise were kept at lower levels, leading to better edge detection. Figure 4 shows segmentation results obtained on this image for the different tested models. The 4DGVF segmentation result (fig. 4e) is the closest to ground truth result (fig. 4a). These observations were confirmed by the Jaccard similarity scores displayed in the middle column of table 1 .

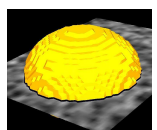

(a)

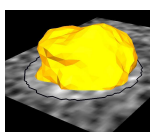

(b)

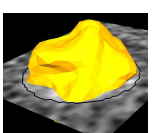

(c)

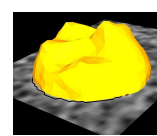

(d)

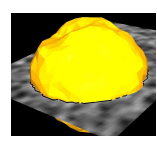

(e)
Fig. 4. Segmentation results for a synthetic $4 \mathrm{D}$ image. (a) ground truth (b) GGVF (c) VFC (d) CGVF (e) 4DGVF.

Table 1. Average Jaccard similarity scores

\begin{tabular}{l|c|c} 
Image & 4D synthetic images & 4D PET simulations \\
\hline \hline GGVF & $0.89 \pm 0.12$ & $0.80 \pm 0.26$ \\
VFC & $0.91 \pm 0.11$ & $0.82 \pm 0.20$ \\
CGVF & $0.81 \pm 0.15$ & $0.81 \pm 0.12$ \\
4DGVF & $\mathbf{0 . 9 5} \pm \mathbf{0 . 1 4}$ & $\mathbf{0 . 8 5} \pm \mathbf{0 . 1 1}$
\end{tabular}

\subsection{Monte Carlo simulations}

We assessed the 4DGVF approach with quantitative results on realistic Monte Carlo simulations of dPET images ge-

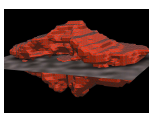

(a)

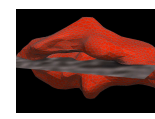

(b)

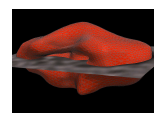

(c)

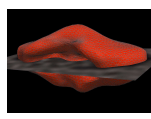

(d)

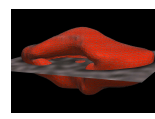

(e)
Fig. 5. Cerebellum segmentation in a $4 \mathrm{D}$ PET simulation. (a) ground truth (b) GGVF (c) VFC (d) CGVF (e) 4DGVF

nerated using GATE, an highly realistic PET image simulator based on the CERN's GEANT4 particles interaction platform [23]. Two 4D images based on the Zubal head phantom [24] were created, which necessitated 90 days of parallel computations on a 12 cores 48 GB RAM computer. For each of the reconstructed images, we studied the segmentation of the cerebellum. Representative segmentation results are shown in Fig. 5. The average quantitative results obtained for the two simulations are displayed in the right column of table 1 . The 4DGVF-based model obtained the best Jaccard similarity in$\operatorname{dex}(0.85)$.

\subsection{Real images}
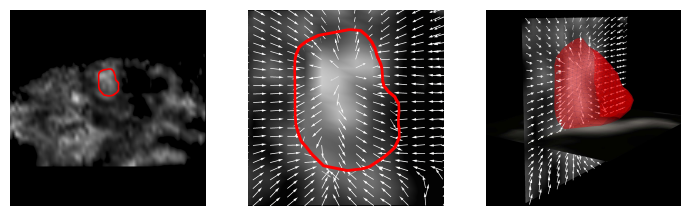

Fig. 6. Left : segmentation of a quinolinic acid lesion in the striatum region of a rat brain. Middle, right : result (in red), superimposed onto the 4DGVF field (in white).

To illustrate the behavior of the 4DGVF method in a real pre clinical context, we performed a dPET acquisition of a rat brain using [18F]-DPA-714, a radiotracer specific to the translocator protein (TSPO). This protein is over expressed under pathologic neuroinflammatory conditions. The inflammation was produced by performing unilateral quinolinic acid lesions in the right striatum of the rat. Figure 6 shows a representative segmentation result for one rat. The shape produced was consistent with the morphology and location of the injured region.

\section{CONCLUSION}

We have proposed a novel external force field in order to perform robust active surface segmentation of 3D volumes in vector-valued images. The proposed method exploits the whole spatio-spectral information available in order to increase its accuracy and reduce its sensitivity to noise. Results on synthetic images as well as real dPET images have confirmed the potentiality of the proposed method for the segmentation of vector-valued images. 


\section{REFERENCES}

[1] M. Kass, A. Witkin, and D. Terzopoulos, "Snakes : Active contour models," International journal of computer vision, vol. 1, no. 4, pp. 321-331, 1988.

[2] A. Dufour, R. Thibeaux, E. Labruyère N. Guillen and J.C. Olivo-Marin "3-D Active Meshes : Fast Discrete Deformable Models for Cell Tracking in 3-D Time-Lapse Microscopy" Image Processing, IEEE Transactions on, vol. 20, no. 7, pp. 1925-1937, 2011.

[3] S. Osher and J.A. Sethian, "Fronts propagating with curvature-dependent speed : algorithms based on hamilton-jacobi formulations," Journal of computational physics, vol. 79, no. 1, pp. 12-49, 1988.

[4] C. Xu and J.L. Prince, "Snakes, shapes, and gradient vector flow," Image Processing, IEEE Transactions on, vol. 7, no. 3, pp. 359-369, 1998.

[5] C. Xu and J.L. Prince, "Generalized gradient vector flow external forces for active contours," Signal Processing, vol. 71, no. 2, pp. 131-139, 1998.

[6] Thomas Brox, Joachim Weickert, Bernhard Burgeth, and Pavel Mrázek, "Nonlinear structure tensors," Image and Vision Computing, vol. 24, no. 1, pp. 41-55, 2006.

[7] S. Di Zenzo, "A note on the gradient of a multi-image," Computer Vision, Graphics, and Image Processing, vol. 33, no. 1, pp. 116-125, 1986.

[8] H.C. Lee and D.R. Cok, "Detecting boundaries in a vector field," Signal Processing, IEEE Transactions on, vol. 39, no. 5, pp. 1181-1194, 1991.

[9] G. Sapiro, "Vector (self) snakes : A geometric framework for color, texture, and multiscale image segmentation," in Image Processing, 1996. Proceedings., International Conference on. IEEE, 1996, vol. 1, pp. 817-820.

[10] X. Xie and M. Mirmehdi, "RAGS : Region-aided geometric snake," Image Processing, IEEE Transactions on, vol. 13, no. 5, pp. 640-652, 2004.

[11] L. Yang, P. Meer, and D.J. Foran, "Unsupervised segmentation based on robust estimation and color active contour models," Information Technology in Biomedicine, IEEE Transactions on, vol. 9, no. 3, pp. 475-486, 2005.

[12] V. Jaouen, P. González, S. Stute, D. Guilloteau, I. Buvat, and C. Tauber, "Vector-based active surfaces for segmentation of dynamic PET images," in Biomedical Imaging (ISBI), 2013 IEEE 10th International Symposium on. IEEE, 2013, pp. 61-64.
[13] B. Li and S.T. Acton, "Active contour external force using vector field convolution for image segmentation," Image Processing, IEEE Transactions on, vol. 16, no. 8, pp. 2096-2106, 2007.

[14] J. Weickert, Anisotropic diffusion in image processing, vol. 1, Teubner Stuttgart, 1998.

[15] A. Cumani, "Edge detection in multispectral images," CVGIP : Graphical models and image processing, vol. 53, no. 1, pp. 40-51, 1991.

[16] G. Sapiro and D.L. Ringach, "Anisotropic diffusion of multivalued images with applications to color filtering," Image Processing, IEEE Transactions on, vol. 5, no. 11, pp. 1582-1586, 1996.

[17] P. Blomgren and T.F. Chan, "Color TV : total variation methods for restoration of vector-valued images," Image Processing, IEEE Transactions on, vol. 7, no. 3, pp. 304-309, 1998.

[18] J. Weickert, "Coherence-enhancing diffusion filtering," International Journal of Computer Vision, vol. 31, no. 2-3, pp. 111-127, 1999.

[19] C. Tauber, H. Batatia, and A. Ayache, "Quasi-automatic initialization for parametric active contours," Pattern Recognition Letters, vol. 31, no. 1, pp. 83-90, 2010.

[20] B. Li and S.T. Acton, "Automatic active model initialization via Poisson inverse gradient," Image Processing, IEEE Transactions on, vol. 17, no. 8, pp. 1406-1420, 2008.

[21] W.E. Lorensen and H.E. Cline, "Marching cubes : A high resolution 3D surface construction algorithm," in ACM Siggraph Computer Graphics. ACM, 1987, vol. 21, pp. 163-169.

[22] P. Jaccard, "Distribution de la flore alpine dans le bassin des Dranses et dans quelques régions voisines," Bulletin de la Société Vaudoise des Sciences Naturelles, vol. 37, pp. 241-272, 1901.

[23] S. Jan et al., "GATE v6 : a major enhancement of the gate simulation platform enabling modelling of CT and radiotherapy," Physics in medicine and biology, vol. 56, no. 4, pp. 881, 2011.

[24] G. Zubal et al., "Computerized three-dimensional segmented human anatomy," Medical Physics-New YorkInstitute of Physics, vol. 21, no. 2, pp. 299-302, 1994. 